\title{
NMR TECHNIQUES APPLIED TO MARTENSITIC TRANSFORMATION
}

\author{
C. DIMITROPOULOS, F. BORSA ${ }^{*, * *}$, S. RUBINI and R. GOTTHARDT \\ Institut de Physique Expérimentale, Ecole Polytechnique Fédérale de Lausanne, CH-1015 Lausanne, \\ Switzerland \\ *Dipartimento di Fisica "A. Volta", Università di Pavia, I-27100 Pavia,Italy \\ ${ }^{* *}$ Department of Physics, Iowa State University, Ames IA 50011,U.S.A.
}

\begin{abstract}
The NMR parameters which should be measured in order to obtain microscopic information about Martensitic Phase Transformations are briefly discussed. Recent results of ${ }^{27} \mathrm{Al}$ and ${ }^{63} \mathrm{Cu} \mathrm{NMR}$ are presented for both ternary alloy $\mathrm{Cu}-\mathrm{Zn}$ - $\mathrm{Al}$ and binary alloys $\mathrm{Cu}-\mathrm{Zn}$ and $\mathrm{Ni}-\mathrm{Al}$. It is shown that the NMR spectrum allows the study of the coexistence of martensite and austenite in the thermoelastic equilibrium range between $M_{S}$ and $M_{F}$ and that the nuclear spin lattice relaxation rate can detect charge density fluctuations at the "soft" interface between the A-phase and the M-phase.
\end{abstract}

\section{1.-INTRODUCTION}

Nuclear Magnetic Resonance (NMR) spectroscopy is a very suitable tool to investigate the local microscopic properties of materials. The nuclei are local probes which are sensitive to interactions within a range of a few lattice cells. However, since the measured parameters result from averages over the given nuclei, the NMR techniques probe the bulk properties of materials, being rather insensitive to surface effects and/or the presence of small $(<1 \%)$ impurity phases. While NMR is a very established method to investigate static and dynamical properties at "reversible" structural phase transitions (SPT)[1], its potential in investigating Martensitic Phase Transitions (MPT's) is not yet fully established[2,3].

The physical significance of the main NMR parameters which will be presented and discussed in the present review are now briefly summarized. Generally speaking, the resonance spectra allow the study of the order parameter, the local atomic arrangement and the local electronic charge density, whereas the relaxation rates $\left(\mathrm{T}_{1}^{-1}, \mathrm{~T}_{2}^{-1}\right)$ are sensitive to low frequency fluctuations which are present at the phase transformations. Since the MPT occurs in metals and in metallic alloys, one of the most important NMR parameter to be measured is the Knight shift, which represent the resonance frequency shift $\delta \nu$ of the NMR line with respect to the Larmor frequency $\nu_{0}$ of the bare nucleus. This shift can be expressed in terms of the local hyperfine field generated at the nuclear site by the conduction electrons:

$$
K=\frac{\delta \nu}{\nu_{0}}=\frac{8 \pi}{3}\left\langle\rho\left(r_{n}\right)\right\rangle_{F} \chi_{P}
$$

Eq. 1 gives an idea of the physical parameters involved in the simplest case of s-character of the conduction electrons: $\chi_{P}$ is the Pauli susceptibility and $\left\langle\rho\left(r_{n}\right)\right\rangle_{F}$ is the charge density at the site of the nucleus in the metal, averaged over the Fermi surface.

The nuclear spin-lattice relaxation rate, $\mathrm{T}_{1}^{-1}$ in metals is mainly due to the scattering of the conduction electrons with the nuclear spins. A simple expression for $\mathrm{T}_{1}^{-1}$ was given by Korringa in the case of s-electrons: 


$$
\left(T_{1} T\right)^{-1}=\frac{4 \pi k_{B}}{\hbar}\left(\frac{\gamma_{N}}{\gamma_{e}}\right)^{2} K(\alpha) K^{2} \propto\left\langle\rho^{2}(r, t)\right\rangle_{F}
$$

where $\gamma_{N}$ and $\gamma_{e}$ are the gyromagnetic ratios of the nucleus and of the electron respectively, and $K(\alpha)$ is a correction factor of order of unity which takes into account electron-electron interactions.

For nuclei with $I>1 / 2$ one can also probe the local electric field gradient (efg) at the nuclear site, via the coupling of the efg tensor with the nuclear quadrupole moment $Q$. The strength of this interaction is measured by the nuclear quadrupole coupling frequency:

$$
\nu_{Q}=\frac{3 e^{2} q Q\left(1-\gamma_{\infty}\right)}{2 I(2 I-1)}
$$

were $e q$ is the greatest component of the efg tensor and $\left(1-\gamma_{\infty}\right)$ is the antishielding factor which depends on the polarizability of the core electrons of the given atom. The quadrupole coupling can modify drastically the NMR spectrum in presence of a local non cubic symmetry $(e q \neq 0)$ and it can introduce an additional mechanism of spin-lattice relaxation although, in metals, the quadrupole contribution to relaxation is generally negligible with respect to the magnetic one described by Eq. 2 .

In the following we are going to present and illustrate some relevant NMR results obtained in alloys undergoing MPT's. The results in the ternary $\mathrm{Cu}-\mathrm{Zn}-\mathrm{Al}$ system are from Ref. 3, to which we refer for details of the data analysis. The results in the binary alloys $\mathrm{Cu}-\mathrm{Zn}$ and $\mathrm{Ni}-\mathrm{Al}$ are preliminary and not yet published.

\section{2.- $\mathrm{Cu}-\mathrm{Zn}-\mathrm{Al}$}

The alloy investigated has the composition $\mathrm{Cu} 66.5 \mathrm{at} \%, \mathrm{Zn} 20.2 \mathrm{at} \%, \mathrm{Al} 13.3 \mathrm{at} \%$, corresponding to $M_{S}=152 \mathrm{~K}, M_{F}=140 \mathrm{~K}, A_{S} \simeq 150 \mathrm{~K}$ and $A_{F} \simeq 165 \mathrm{~K}$ as determined by Differential Scanning Calorimetry (DSC) measurements. The sample used for NMR work consisted of small pieces $(2 \times 4 \times 0.3 \mathrm{~mm})$ cut from the original ingot by means of a spark machine, and was cycled twenty times between room temperature and liquid nitrogen temperature in order to stabilize the transformation and the hysteresis loop.

The ${ }^{27} \mathrm{Al}(I=5 / 2)$ absorption NMR spectrum is composed by a narrow $(4 \mathrm{kHz})$ unperturbed central line $(1 / 2,-1 / 2$ transition) superimposed to a broad (about $50 \mathrm{kHz}$ ) line corresponding to the satellite transitions, subjected to a distribution of local efg's due to atomic disorder and/or defects. The Knight shift increases by about $9 \%$ on going from the austenite to the martensite, as shown in Fig. 1.

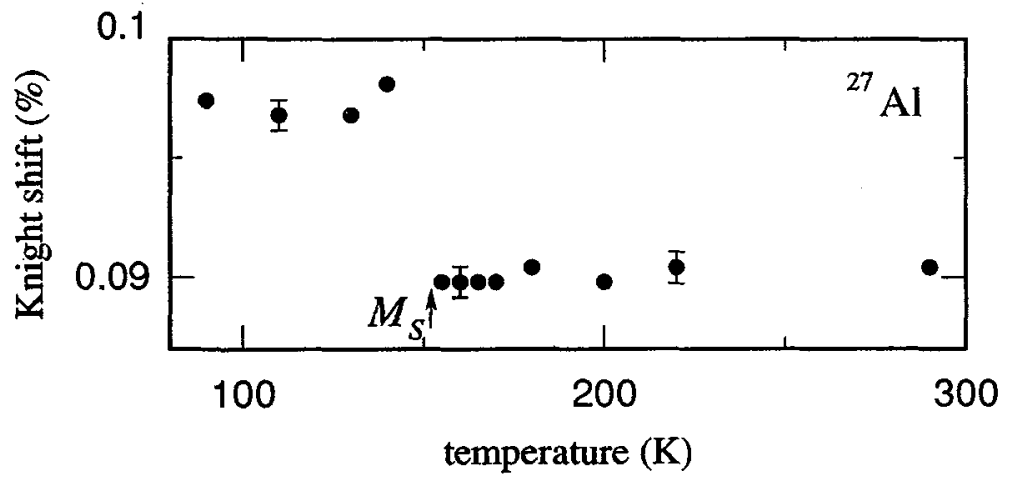

Figure 1. ${ }^{27} \mathrm{Al}$ Knight shift vs temperature at $\mathrm{H}_{0}=7 \mathrm{~T}$ in $\mathrm{Cu}-\mathrm{Zn}-\mathrm{Al}$.

In the temperature region between $M_{S}$ and $M_{F}$ the ${ }^{27} \mathrm{Al}$ NMR line is the superposition of two lines, centered at the resonance frequency of the A-phase and the M-phase respectively (Fig. 2(a)). 
By analyzing the evolution of the spectra as a function of temperature with the aid of a computer fitting scheme, one can obtain the evolution of the nucleation and the growth of the martensite in terms of the fraction of the M-phase present, as shown in Fig. 2(b).
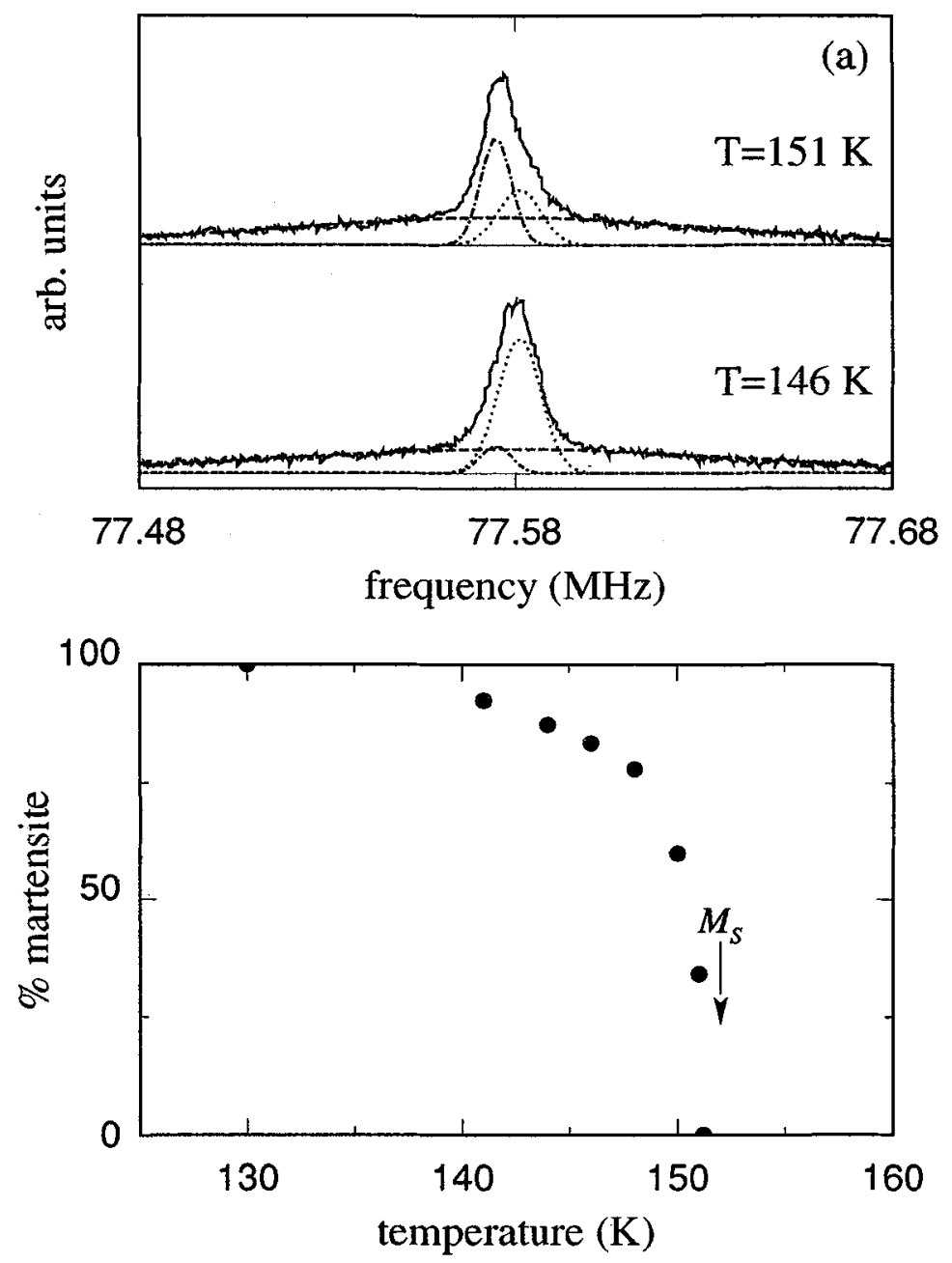

Figure 2. (a) ${ }^{27} \mathrm{Al}$ NMR line in $\mathrm{H}_{0}=7 \mathrm{~T}$, showing the superposition of the two signals originating from the austenite (dash-dotted line) and the martensite(dashed line), in the coexistence temperature range. (b) Temperature dependence of the fraction of martensite present between $M_{S}$ and $M_{F}$ as obtained from the analysis of the NMR line shown in Fig. 2(a).

From Fig. 2(b) it appears that the nucleation of the martensite is rather "explosive" as expected for a strongly first order phase transition. The amount of martensite present at any temperature between $M_{S}$ and $M_{F}$ was found to be time independent within the limit of the experimental error, as expected for a true thermoelastic equilibrium phase. Since two distinct NMR signals are present between $M_{S}$ and $M_{F}$, the lifetime of the martensitic regions must be longer than the inverse of the frequency separation between the two NMR lines, i.e. $\tau \geq 10^{-4} \mathrm{~s}$.

A small anomaly is observed in the $\mathrm{T}_{1}^{-1}$ of both ${ }^{27} \mathrm{Al}$ and ${ }^{63} \mathrm{Cu}$ nuclei in the temperature region between $M_{S}$ and $M_{F}$, as shown in Fig. 3 . 
Outside the transition region, $\mathrm{T}_{1}^{-1}$ decrease linearly with $\mathrm{T}$ as expected from Eq. 2 . The anomaly observed is ascribed to large charge density fluctuations at the boundary between the martensitic and the austenitic phase. In fact, since the Knight shift is proportional to the time average charge density (see Eq. 1) while the $\mathrm{T}_{1}^{-1}$ is proportional to the average of the square of the charge density (see Eq. 2), one expects an extra contribution to the spin lattice relaxation rate of the order of $\frac{\left\langle\delta \rho^{2}\right\rangle}{\rho_{0}^{2}}$ in presence of local charge density fluctuation $\delta \rho$. Thus, it appears that below $M_{S}$ large amplitude atomic motions occur at the interfaces between the "hard" martensite and the "soft" austenite.
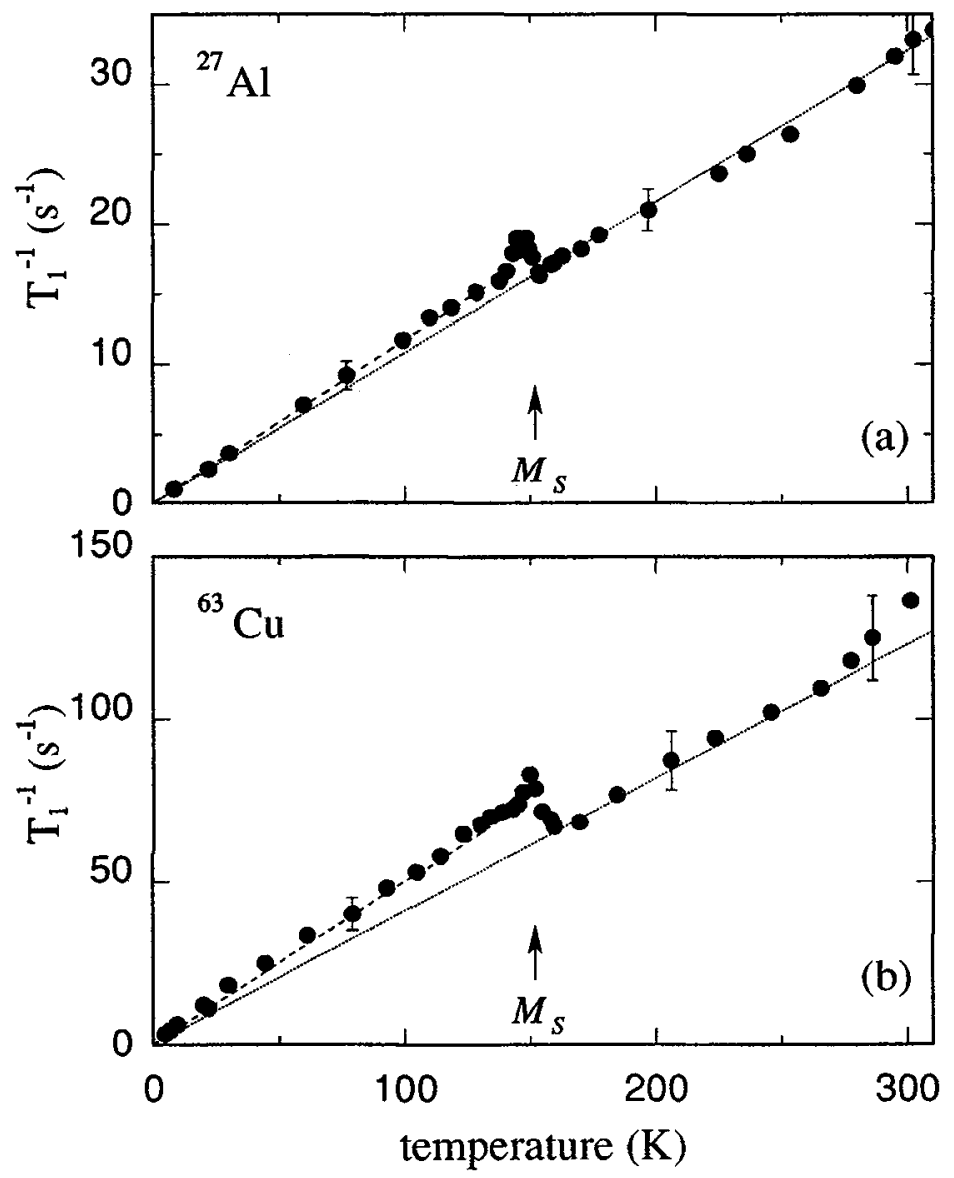

Figure 3. Nuclear spin-lattice relaxation rate $\mathrm{T}_{1}^{-1}$ vs temperature in $\mathrm{Cu}-\mathrm{Zn}$-Al alloy: (a) ${ }^{27} \mathrm{Al}$, (b) ${ }^{63} \mathrm{Cu}$.

The search for precursor effects above $M_{S}$ in both ${ }^{27} \mathrm{Al}$ and ${ }^{63} \mathrm{Cu}$ spectra and relaxation rates was unsuccessful. It is noted that long wavelength static modulation or embryonic fluctuations would have a negligible effect on the ${ }^{27} \mathrm{Al}$ and ${ }^{63} \mathrm{Cu}$ spectra because the local distortion around the nuclear sites remains small. On the other hand, quadrupole contributions to relaxation by fast $\left(\tau \geq 10^{-11} \mathrm{~s}\right.$ ) heterophase or embryonic fluctuations would be masked by the dominant magnetic relaxation mechanism. 


\section{3.- $\mathrm{Cu}-\mathrm{Zn}$ and $\dot{\mathrm{Ni}}-\mathrm{Al}$}

The binary $\mathrm{Cu}-\mathrm{Zn}$ alloy investigated has a composition $\mathrm{Cu} 60 \mathrm{at} \%$, $\mathrm{Zn} 40 \mathrm{at} \%$, with $M_{S}=181 \mathrm{~K}$, $M_{F}=153 \mathrm{~K}, A_{S} \simeq 178 \mathrm{~K}$ and $A_{F} \simeq 200 \mathrm{~K}$ as determined from the DSC measurements. The NMR sample consisted of small pieces $(0.3 \times 0.3 \times 0.3 \mathrm{~mm})$ cut from a $\beta$ phase single crystal by means of a wire saw, and was cycled twenty times between room temperature and liquid nitrogen temperature.

The ${ }^{63} \mathrm{Cu}$ NMR line shape and width are similar to those observed in the $\mathrm{Cu}-\mathrm{Zn}-\mathrm{Al}$ alloy [3]. The second order quadrupole effects dominate the broadening of the NMR spectra in both phases, and the average Knight shift increases by about $10 \%$ on going trough the transition.

Contrary to the results in the ternary $\mathrm{Cu}-\mathrm{Zn}$ - $\mathrm{Al}$ alloy, the ${ }^{63} \mathrm{Cu} \mathrm{T}_{1}^{-1}$ vs $\mathrm{T}$ shows no anomaly in the temperature region between $M_{S}$ and $M_{F}$ (see Fig. 3(a)).
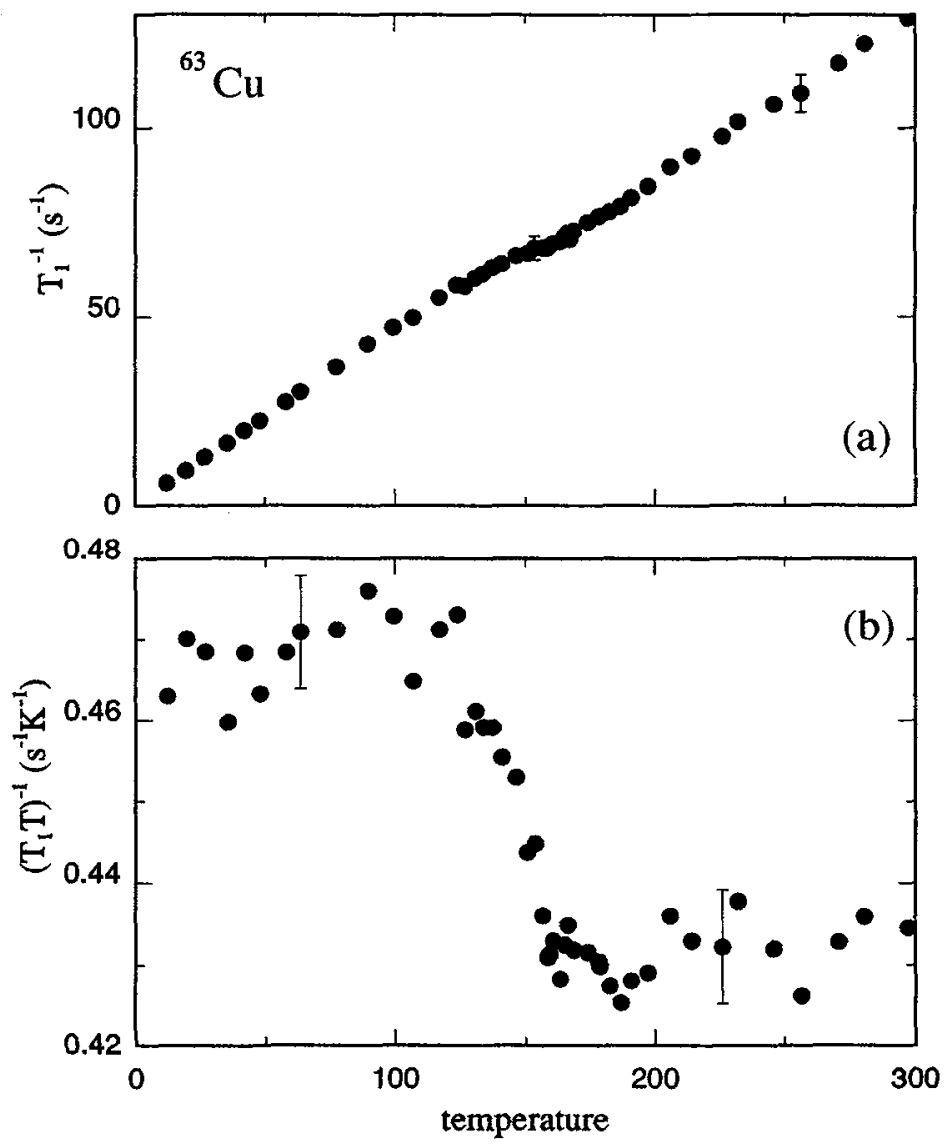

Figure 3. (a) ${ }^{63} \mathrm{Cu} \mathrm{T}_{1}^{-1}$ versus temperature in $\mathrm{Cu}-\mathrm{Zn}$ alloy, (b) the quantity $\left(T_{1} T\right)^{-1}$ plotted vs temperature.

The $\mathrm{T}_{1}^{-1}$ vs $\mathrm{T}$ curve is a straight line as predicted by Eq. 2 with a different slope in the austenite and the martensite respectively (see Fig. 3(a)). The plot of $\left(T_{1} T\right)^{-1}$ vs $\mathrm{T}$ shown in Fig. 3(b)) put in evidence the discontinuity in the transition region, which is related to the change of Knight shift (see Eq. 2).

The two $\mathrm{Ni}-\mathrm{Al}$ alloys investigated have composition $\mathrm{Ni} 62$ at\%, $\mathrm{Al} \mathrm{38at \%}$ and $\mathrm{Ni} 63 \mathrm{at} \%, \mathrm{Al} 37$ at $\%$ respectively. Special care was take to homogenize the alloy at $1300 \mathrm{~K}$ for one week. The samples were 
then crushed in a mortar to obtain the desired particle size. For each composition two samples were prepared, one with approximate particle size of $0.5 \div 1 \mathrm{~mm}$, and the other with size less then $150 \mu \mathrm{m}$. Part of the sample was annealed at $800 \mathrm{~K}$ for 15 hours to relieve strains. No difference in the behavior of the MPT was observed by NMR between the annealed and the unannealed samples. In the case of the $\mathrm{Ni}$ - Al alloys the $\mathrm{DSC}$ yields very weak signals, from which one can estimate approximatively the transition temperatures only for the Al-37at\% sample: $M_{S} \simeq 290 \mathrm{~K}, M_{F} \simeq 260 \mathrm{~K}, A_{S} \simeq 270 \mathrm{~K}$ and $A_{F} \simeq 300 \mathrm{~K}$.

The ${ }^{27} \mathrm{Al} \mathrm{NMR}$ line in $\mathrm{Ni}-\mathrm{Al}$ is about five times broader then the ${ }^{27} \mathrm{Al}$ line in $\mathrm{Cu}-\mathrm{Zn}-\mathrm{Al}$. The additional inhomogeneous broadening is of magnetic origin, and should probably be ascribed to a distribution of Knight shift values at the $\mathrm{Al}$ site, due to the random second nearest neighbour local environment in the CsCl-type $\beta$-phase of the alloy.

The Knight shift vs $\mathrm{T}$ varies smoothly as one goes through the MPT as shown in Fig. 4.
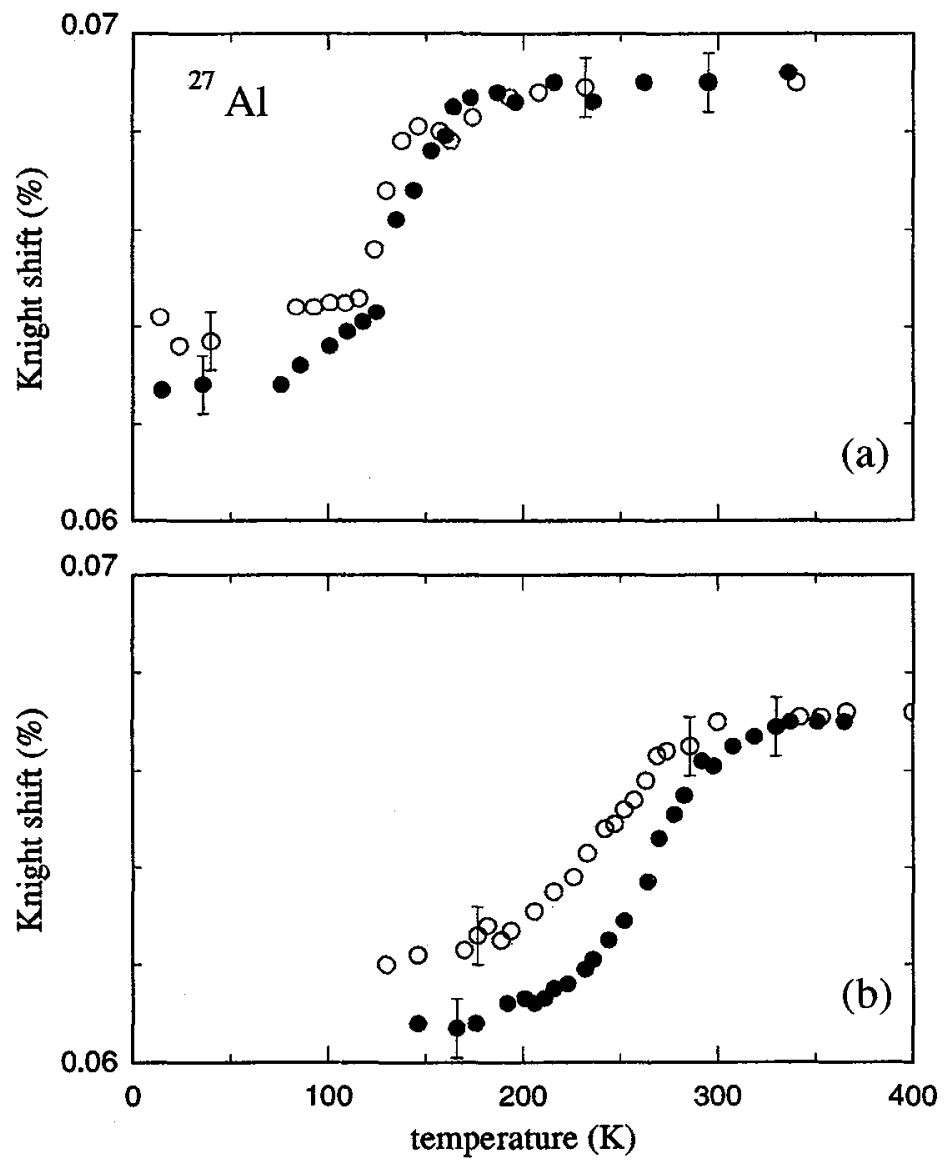

Figure 4: ${ }^{27} \mathrm{Al} \mathrm{Knight} \mathrm{shift} \mathrm{vs} \mathrm{temperature} \mathrm{at} \mathrm{H}_{0}=8 \mathrm{~T}$ in $\mathrm{Ni}$-Al alloys for samples with different particle sizes: $\bullet 0.5 \div 1 \mathrm{~mm}, \circ<150 \mu \mathrm{m}$. (a) 38 at\% $\mathrm{Al}$, (b) 37 at $\% \mathrm{Al}$.

One remarkable feature is that the transition temperature $M_{S}$ is lowered by several degrees when the sample is reduced in the form of a fine powder but at the same time the width of the transition region $M_{S}-M_{F}$ remains the same or even decreases (see Fig. 4). It is noted that by comparing the Knight shift of the particles of different size in Fig. 4, one can infer that in the small particles sample 
a fraction of austenite remains untransformed even at low temperature. This behavior is even more pronounced in $\mathrm{Cu}-\mathrm{Zn}$ alloy, as observed by neutron scattering measurements[4].

The ${ }^{27} \mathrm{Al} \mathrm{T}_{1}^{-1}$ in the Al-38at\% sample shown in Fig. 5 varies linearly with $\mathrm{T}$ according to Eq. 2, with a slightly different slope in the austenite and the martensite, reflecting the difference of Knight shift between the two phases, but without any detectable anomaly in the transition region.
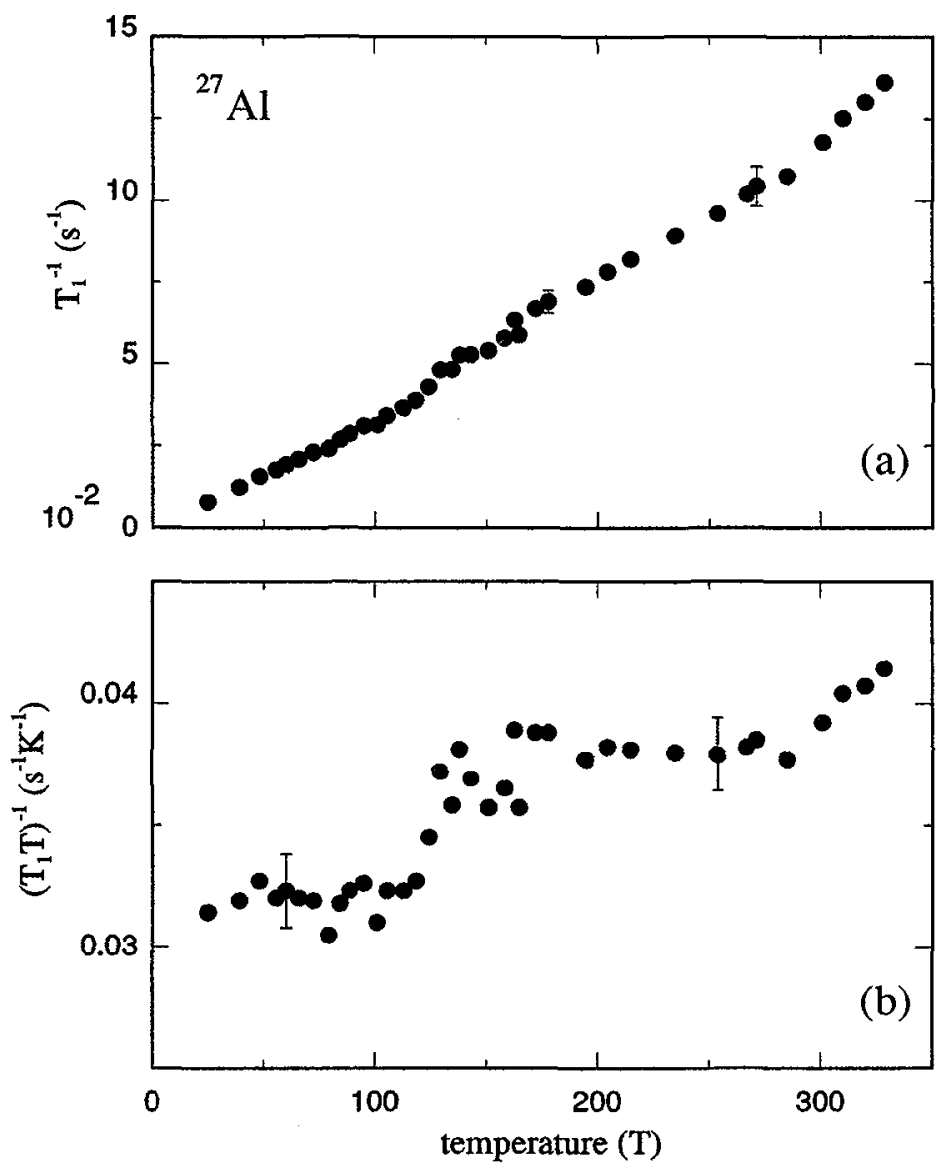

Figure 5:(a) ${ }^{27} \mathrm{Al}, \mathrm{T}_{1}^{-1}$ vs temperature at $\mathrm{H}_{0}=7 \mathrm{~T}$ in Ni-38 at \%Al. (b) the quantity $\left(T_{1} T\right)^{-1}$ plotted vs temperature.

A special effort was made in order to analyze the ${ }^{27} \mathrm{Al}$ NMR spectrum in the temperature interval between $M_{S}$ and $M_{F}$. No asymmetry of the line comparable to the one observed in $\mathrm{Cu}-\mathrm{Zn}$ - $\mathrm{Al}$ alloy (see Fig. 2(b)) can be observed in $\mathrm{Ni}$-Al alloys, although some detectable broadening is present (see Fig. 6).

An unambiguous analysis of the data is prevented by the lack of resolution due to the fact that the ratio between the maximum observed frequency shift and the half-width of the line is less than one. The fraction of martensite in the thermoelastic equilibrium region (Fig. 7) has been tentatively evaluated by fitting the NMR line with two gaussian.

In order to get a good fit we had to assume that one line is centered at the resonance frequency of the austenite, with a line width which varies with temperature as the background broadening indicated by the straight line in Fig. 6 ; the second line, belonging to the martensite, was centered at the a resonance frequency which varies linearly with the fraction of martensite shown in Fig. 7 . 


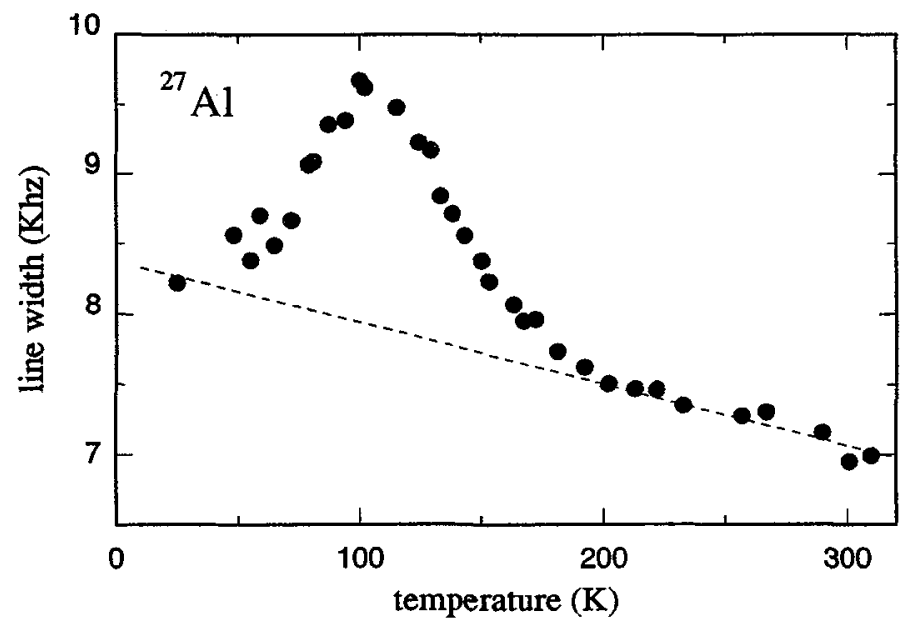

Figure $6 .{ }^{27} \mathrm{Al}$ line width of the central transition $(+1 / 2,-1 / 2)$ vs temperature at $\mathrm{H}_{0}=7 \mathrm{~T}$ in NI 38 at $\%$ Al. The data were obtained by fitting the Fourier transform of the NMR free precession decay with a gaussian after subtracting off the contribution due to the satellites. The straight line is a guide to the eye which is assumed to give the temperature dependence of the "background" magnetic inhomogeneous broadening.

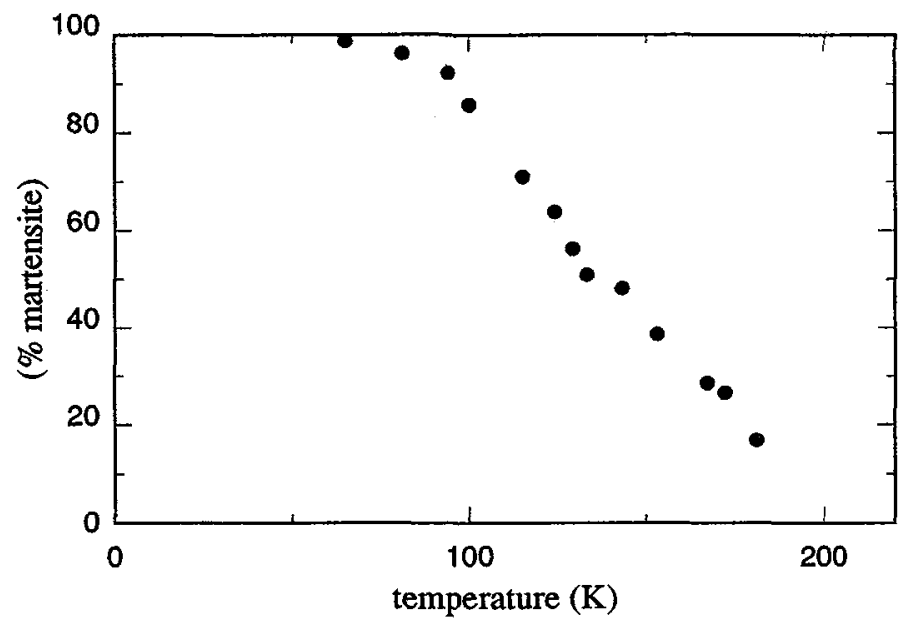

Figure 7. Fraction of martensite coexisting with the austenite vs temperature for $\mathrm{Ni}-38 \mathrm{at} \% \mathrm{Al}$ alloy, as deduced from the computer fit of the NMR line as described in the text. 


\section{4.- Conclusion}

The NMR effects observed up to now at the MPT in $\mathrm{Cu}-\mathrm{Zn}-\mathrm{Al}, \mathrm{Cu}-\mathrm{Zn}$ and $\mathrm{Ni}-\mathrm{Al}$ alloys are small and difficult to interpret. Nevertheless, a few encouraging results have been obtained. Firstly, the Knight shift change between the austenite and the martensite is well defined, even when the transition is broad and close to second order, as indicated by the lack of calorimetric effects in the DSC. This should allow one to study changes of $M_{S}$ and of the width of the transition in samples of different size and as a function of pressure and other physical parameter. Secondly, a small anomaly in $\mathrm{T}_{1}^{-1}$ can be observed in the ternary $\mathrm{Cu}-\mathrm{Zn}-\mathrm{Al}$ alloy but not in the binary $\mathrm{Cu}-\mathrm{Zn}$ and $\mathrm{Ni}-\mathrm{Al}$ alloys. Although the causes of this different behaviour is not presently understood, it could be very well correlated with the differences in the nucleation and growth process of the martensite in strongly first-order transition (e.g. $\mathrm{Cu}-\mathrm{Zn}-\mathrm{Al}$ ) with respect to weak first-order ones (e.g. Ni-Al). The most promising NMR application to the study of MPT's appears to be the measurement of the fraction and of the degree of deformation of the martensitic phase in the temperature region $M_{S^{-}} M_{F}$, through the analysis of the amplitude and position and width of coexisting NMR lines. This kind of analysis has put in evidence a different behavior of the nucleation and growth process in the ternary alloys $\mathrm{Cu}-\mathrm{Zn}-\mathrm{Al}$ with respect to $\mathrm{Ni}-\mathrm{Al}$ alloys. While in the first case the nucleation is "explosive" at $M_{S}$ and the two phases coexists in the temperature interval $M_{S}-M_{F}$ with the martensite in its final equilibrium lattice deformation state, in the second case the nucleation appears to proceed gradually in a temperature range of $100 \mathrm{~K}$. Furthermore, it appears that the lattice deformation of the martensite increases from $M_{S}$ to the final value reached at $M_{F}$ and below, and it is not uniform in the interval $M_{S}-M_{F}$, as evidenced by the broadening of the NMR line (see Fig. 6). These tentative conclusion should be confirmed by measurements in systems where narrow NMR lines with large Knight shift are present. Good candidates in this respect are the $\mathrm{Cd}-\mathrm{Ag}$ and $\mathrm{Cd}-\mathrm{Au}$ alloys which are presently under investigation.

\section{Acknowledgements}

Thanks are due to J.Ziolo and D.R.Torgeson for the collaboration in the experiments performed in Ni-Al alloys.

\section{REFERENCES}

/1/ Rigamonti,A.: Adv. in Phys. $\underline{33}, 1984,115$

/2/ S.Rubini, C.Dimitropoulos, R.Gotthardt and F.Borsa, Helv. Phys. Acta $\underline{62}, 1989,780$

/3/ S.Rubini, C.Dimitropoulos, R.Gotthardt and F.Borsa, Phys. Rev B, 44 (1 August 1991)

/4/ M.Zölliker, private communication. 